\title{
Tratamento conservador da cárie oclusal e proximal: novas possibilidades
}

\author{
Conservative treatment of occlusal and proximal caries: new possibilities
}

Tratamiento conservador de la caries oclusal y proximal: nuevas posibilidades

Leonardo Castro Ribeiro Leite ${ }^{1}$, Madson Henrique Araújo Feitoza Faustino ${ }^{1}$, Clécio Tenório de Azevedo ${ }^{1}$, Izabel Cristina Gomes de Mendonça ${ }^{1 *}$.

\section{RESUMO}

Objetivo: Buscar na literatura científica, evidências pertinentes aos princípios do tratamento conservador da cárie, com ênfase nas faces oclusais e proximais e demonstrar sua eficiência na prática clínica. Revisão bibliográfica: Mesmo com muitos obstáculos no diagnóstico de cáries incipientes dos dentes, e com todo aparato tecnológico disponível, a prática de uso de mecanismos não operatórios, com o início da remoção mecânica tão bem como selantes em fissuras e estruturas retentoras de biofilme, e aplicação tópica de flúor se comprovam na eficácia terapêutica no tratamento de lesões cariosas ativas em esmalte. A dificuldade em desconstruir décadas de condutas convencionais para tratamento de cáries incipientes em faces oclusais e proximais, confronta o que se é doutrinado nas universidades, levando o profissional a se sentir inseguro ao aplicar técnicas conservadoras, não se limitando a área da lesão e a consequentemente a perda de tecido sadio. Considerações finais: Os estudos citados nesse trabalho sustentam que é possível diminuir a chance de desenvolvimento de cárie em dentes recém-erupcionados ou contê-la. Nas lesões proximais a prioridade é impedir a ocorrência e progressão da lesão.

Palavras-chave: Tratamento conservador, Cárie dentária, Selantes dentários, Flúor.

\begin{abstract}
Objective: To search the scientific literature for pertinente evidence to the principles of conservative treatment of caries, with emphasis on the occlusal and proximal surfaces and to demonstrate its efficiency in clinical practice. Bibliographic review: Even with many obstacles in the diagnosis of incipient tooth decay, and with all available technological apparatus, the practice of using non-operative mechanisms, with the beginning of mechanical removal as well as sealants in fissures and biofilm retaining structures, and topical application of fluoride are proven in the therapeutic efficacy in the treatment of active carious enamel lesions. The difficulty in deconstructing decades of conventional procedures for the treatment of incipiente caries on occlusal and proximal faces, confronts what is indoctrinated in universities, leading the professional to feel insecure when applying conservative techniques, not limited to the area of the lesion and consequently the loss of healthy tissue. Final considerations: The studies cited in this work maintain that it is possible to decrease the chance of developing caries in newly erupted teeth or to contain it. In proximal injuries, the priority is to prevent the occurrence and progression of the injury.
\end{abstract}

Keywords: Conservative treatment, Dental caries, Dental sealants, Fluoride.

\section{RESUMEN}

Objetivo: Buscar en la literatura científica, evidencias pertenientes a los principios del tratamiento conservador de las caries, con énfasis en las superficies oclusales y proximales y demostrar su eficiencia en la práctica clínica. Revisión bibliográfica: Aun con muchos obstáculos en la diagnosis de caries incipientes en los dientes, y con todo aparato tecnológico disponible, la práctica de uso de mecanismos no operatórios, con el inicio de remoción mecánica tan bien como sellador en hendiduras y estructuras que atrapen la biopelícula, y aplicación tópica de flúor se comproban en la eficiencia terapéutica en el tratamiento de lesiones por caries activas en el esmalte dental. La dificultad en deconstruir décadas de conductas convencionales para el tratamiento de caries incipientes en superficies oclusales y proximales, confronta a lo que es

${ }^{1}$ Centro Universitário Cesmac (CESMAC), Maceió - AL. *E-mail: belgomess@gmail.com 
adoctrinado en las universidades, llevando al profesional a sentirse inseguro cuando aplica técnicas conservadoras, no centrándose en el área de la lesión y la consequentemente pérdida de tejido sano. Consideraciones finales: Los estudios mencionados en este trabajo sostienen que es posible disminuir la posibilidad del desarrollo de dientes recién erupcionados o contenerla. En las lesiones proximales la prioridad es evitar la aparición y progresión de la lesión.

Palabras clave: Tratamiento conservador, Caries dental, Selladores dentales, Flúor.

\section{INTRODUÇÃO}

A cárie dental é uma das doenças mais antigas que acomete a cavidade oral. Caracteriza-se pela dissolução dos tecidos dentários sob a ação de ácidos provenientes da fermentação dos alimentos, em particular os carboidratos, por bactérias acidogênicas presentes na microbiota normal da boca (SILVA EL, et al., 2017). A etiologia da cárie é relativamente complexa porque envolve vários fatores que, agindo conjuntamente e sem interrupção, promovem a destruição lenta e progressiva do dente, entre eles destacamse a dieta, o hospedeiro, a saliva e o tempo de permanência deles na superfície do dente. Fatores estes que, quando devidamente controlados, interrompem o curso da doença constituindo dessa forma os quatro pilares nos quais se baseiam o tratamento preventivo e o controle da mesma (SILVA BP, et al., 2018; FEJERSKOV O, et al., 2015).

Sem o devido controle, a dissolução progride e deixa sequelas na superfície dentária em forma de cavidades que se convencionou chamar de lesões cariosas. O tratamento convencional dessas lesões, usado por décadas como a terapêutica predominante, consiste na remoção de todo tecido comprometido, com o intuito de deixar uma base de tecido hígido e endurecido sobre o qual o material restaurador é inserido (RIBEIRO AA, 2016). Contudo, esse tratamento muitas vezes fragiliza a estrutura remanescente, aumentando as chances de fratura tanto do dente quanto da restauração, levando a constantes substituições dessas restaurações que vão se tornando cada vez mais complexas culminando com a perda do elemento dental (BATISTA MJ, et al., 2015).

A constatação da falência desse método como tratamento definitivo da cárie aliado a uma maior compreensão tanto da etiologia quanto da progressão da doença nos tecidos dentários, contribuiu decisivamente para uma mudança significativa na filosofia de tratamento das lesões cariosas, ao mesmo tempo em que a evolução dos materiais odontológicos passou a permitir restaurações mais conservadoras (TUMENAS I, et al., 2014). Essa nova filosofia de tratamento passou a priorizar abordagens mais conservadoras e menos invasivas, culminando com o advento da mínima intervenção na Odontologia, a qual envolve técnicas de promoção de saúde e prevenção, para impedir a instalação da lesão, e a adoção de condutas clínicas na paralização do processo carioso e controle da evolução em lesões incipientes (TUMENAS I, et al., 2014; FEJERSKOV O e KIDD E, 2011).

Mesmo com um declínio significativo na prevalência de cárie desde a década de 1970, o manejo das cáries oclusais e proximais continua sendo um importante desafio, principalmente no que concerne à adoção das condutas minimamente invasivas as quais fogem do contexto tradicionalmente empregado há anos no tratamento dessa doença e nesse sentido, a resiliência do profissionais é muito grande, até porque, o tratamento tradicional, doutrinado nas diversas academias preconizando a remoção cirúrgica de todo o tecido cariado ainda em esmalte, foi por muito tempo a única conduta aceita no tratamento da cárie (CARVALHO JC, et al., 2016; RIBEIRO AA, 2016).

As terapêuticas não invasivas buscam reduzir a atividade de cárie com o controle do biofilme e aplicação de flúor, quando o substrato dentário ainda se encontra em atividade inicial de cárie, enquanto que, nos casos em que a lesão cariosa já estiver estabelecida, recomenda-se optar por tratamentos minimamente invasivos ou também micro invasivos, que vão desde a aplicação de selantes até a infiltração de materiais restauradores, construindo uma barreira mecânica para os agentes cariogênicos e impedindo a evolução da doença (SPLIETH CH, et al., 2020; MENDONÇA ICG, et al., 2019).

Diante desse contexto, este artigo tem por objetivo buscar na literatura científica, evidências pertinentes aos princípios do tratamento conservador da cárie, com ênfase nas faces oclusais e proximais e demonstrar sua eficiência e aplicabilidade na prática clínica. 


\section{REVISÃO BIBLIOGRÁFICA}

\section{Tratamento conservador da cárie oclusal em esmalte}

Em um passado recente, a cárie era ensinada nas faculdades como uma doença de progressão rápida e irreversível, que só poderia ser tratada com a restauração do dente afetado. Só no início dos anos 1980, com um maior conhecimento da sua etiologia, evolução e controle, bem como dos benefícios do flúor e evolução dos materiais adesivos, é que teve início uma abordagem mais preventiva e menos operatória para seu tratamento. Daí por diante, o conceito de que a cárie deve ser controlada usando métodos preventivos não invasivos, tanto quanto possível, já é comumente aceito principalmente pelos profissionais mais jovens (LASKE M, et al., 2019).

O grande obstáculo na tomada de decisão para o tratamento das lesões de cárie incipientes na face oclusal é o diagnóstico preciso. De uma maneira geral, o exame radiográfico é o mais empregado na pesquisa de cárie. No entanto, em se tratando de lesões oclusais apenas no esmalte, o método apresenta baixa sensibilidade sendo mais eficaz quando usado como exame complementar em lesões proximais, já que as lesões de cárie iniciais não penetraram o suficiente na dentina oclusal para que sua desmineralização seja evidente radiograficamente (LASK M, et al., 2019; TAGLIFERRO E, et al., 2019; FEJERSKOV O e KIDD E, 2007).

O emprego de exploradores afiados, além de provocar danos à superfície do dente, pode levar a um falso diagnóstico por se prender nas fóssulas e fissuras hígidas inerente à anatomia dentária (TAGLIFERRO E, et al., 2019). Soares GG, et al. (2012), ao compararem métodos avançados de detecção de cárie como Transiluminação por Fibra Óptica (FOTI), Quantificação da Fluorescência induzida por Luz (QLF), Medição de Condutância Elétrica "Electrical Conductance Measurement" (ECM), concluíram que eles até permitem detectar lesões iniciais, mas sempre em dentina, e o profissional precisa ter domínio desses métodos para avaliar os resultados.

O método de inspeção visual-tátil é o elencado entre os pesquisadores como sendo o que apresenta melhores resultados na detecção das lesões, sem danificar a superfície examinada. Para isso o dente precisa estar limpo e seco para observar melhor tanto a textura da lesão como a topografia da superfície examinada. A rapidez e facilidade desse método o elegeu como a melhor escolha para o diagnóstico de cárie, principalmente quando é associado à avaliação da atividade da lesão (TAGLIFERRO E, et al., 2019; NYVAD B e BAELUM V, 2018; NYVAD B, et al., 2011).

No que tange à atividade da lesão cariosa ela pode ser considerada ativa ou inativa, a diferenciação entre elas se concentra no aspecto clínico e leva em consideração a textura e a integridade da superfície observada. Desse modo, as lesões de cárie em esmalte não cavitadas e ativas têm uma superfície opaca esbranquiçada/amarelada, com aparência de giz ou branco-neon, devido à dispersão de luz, e a superfície parece áspera quando uma sonda, sem ponta afiada, é movida sobre ela. Ao passo que, as lesões não cavitadas inativas são brilhantes, devido à reflexão da luz, e podem variar a cor do branco, marrom ou preto, e parecem lisas ao passar a sonda suavemente sobre elas. É importante destacar que uma lesão inativa ou interrompida tem menor probabilidade de progressão do que uma lesão ativa (NYVAD B e BAELUM V, 2018; MAKHIJA SK, et al., 2018).

Do ponto de vista estrutural, o esmalte é um sólido microporoso com espaços intercristalinos ocupados por água e material orgânico (proteínas relativas ao desenvolvimento deixadas durante a maturação do esmalte). Os cristais do esmalte são compostos por hidroxiapatita, fluorapatita e outros componentes sólidos. A saliva tem importante papel no processo de manutenção dos minerais do esmalte. Sob condições fisiológicas normais, ela se mantém supersaturada em relação à hidroxiapatita e a fluorapatita por conter consideráveis quantidades de cálcio e fosfato em sua composição, além de eletrólitos e moléculas orgânicas que contribuem para manter o pH da boca alcalino. Quando o pH se torna ácido, após a ingestão de alimentos, por exemplo, a saliva se torna subsaturada em relação ao esmalte e ocorre uma precipitação dos cristais de esmalte para o meio oral, portanto, a força que impulsiona a dissolução é o grau de subsaturação salivar. Contudo, quando a acidez é controlada pela capacidade tampão da saliva e o biofilme é removido mecanicamente, a saturação se estabiliza e os cristais parcialmente desmineralizados são incorporados ao esmalte (FEJERSKOV O, et al., 2015). 
No que se refere à instalação da cárie, o fator preponderante é a presença da placa bacteriana (biofilme) na qual o produto ácido do metabolismo das bactérias cariogênicas, influenciado pela dieta rica em carboidratos, inicia uma lesão ativa de cárie em aproximadamente 4 semanas de exposição a ele, sem nenhum tipo de intervenção. Isso indica que a remoção mecânica do biofilme é imprescindível para o controle da lesão (FEJERSKOV O, et al., 2007).

O flúor é também um importante coadjuvante nesse processo, pois sua presença torna a hidroxiapatita menos solúvel e reduz drasticamente a quantidade de cálcio que pode ser liberado do esmalte em condição ácida. Ele interfere no desenvolvimento da lesão de tal forma que chega a controlar, e até mesmo paralisar a progressão dela, isso acontece pela capacidade que ele tem de interferir no processo de desmineralização, por se incorporar aos cristais de fluorapatita, ao mesmo tempo em que aumenta a velocidade de remineralização. Portanto, o flúor pode ser usado como agente terapêutico que reduz a taxa de desmineralização e aumenta a incorporação mineral à superfície atingida. Os dentifrícios com flúor são o melhor método de disponibilização de flúor desenvolvido por ser relativamente barato e atingir uma grande parcela da população (GAO SS, et al., 2016; FEJERSKOV O e KIDD E, 2007).

Por conseguinte, o primeiro passo para o controle da progressão da lesão é a remoção do biofilme, seguido do controle da dieta e do uso do flúor do dentifrício como agente terapêutico interferindo no processo de desmineralização e remineralização das lesões iniciais além de um possível efeito na acidogenicidade e cariogenicidade da placa que lhe é atribuído (FEJERSKOV O e KIDD E, 2007).

Há um consenso entre os estudiosos que as fissuras oclusais do primeiro molar permanente são geralmente os primeiros sítios a desenvolver cáries (GORDAN VV, et al., 2010; HASSAN AM e MOHAMMED SG, 2019; PETRIK JA, et al., 2020). A razão para isso é, indubitavelmente, a anatomia dessa superfície que favorece a retenção de biofilme nos sulcos e fossas como também o tempo de erupção relativamente longo, impedindo a limpeza mecânica pelo atrito com o antagonista e dificultando a escovação, levando a uma incidência maior de lesões ativas em comparação com os dentes já totalmente erupcionados (CARVALHO JC, et al., 2016). Os primeiros molares permanentes são considerados elementos dentais de extrema importância para o sistema estomatognático (PETRIK JA, 2020).

Sendo assim, após todas as evidências mostradas até aqui, a face oclusal dos molares é a área mais crítica na decisão sobre o tratamento da cárie dentária. A terapia não cirúrgica (flúor, selante) é recomendada quando o processo carioso está confinado ao esmalte e quando as lesões dentinárias estão inativas. Enquanto que a abordagem cirúrgica é reservada para lesões ativas que penetraram na dentina (GORDON VV, et al., 2010). Os tratamentos mais indicados para lesões de cárie oclusal incipientes é o selamento com resina ou ionômero e aplicação tópica de flúor (gel ou verniz) (CHESTNUTT IG, et al., 2017).

Um trabalho comparando a eficácia clínica de selante de fissuras e verniz fluoretado na prevenção de cárie dentária em primeiros molares permanentes foi realizado por Chestnutt IG, et al. (2017). Os achados do estudo mostraram que a aplicação semestral de verniz fluoretado resultou em prevenção de cárie, mas que não foi significativamente diferente daquela obtida pela aplicação e manutenção de selante de fissura após 36 meses. Já a avaliação do custo-efetividade de um único selamento com ionômero de vidro modificado por resina e de um verniz fluoretado, aplicado semestralmente, durante 24 meses, concluiu que uma única aplicação de selante foi a intervenção com maior custo-efetivo (TAGLIAFERRO E, et al., 2013).

Ao confrontar durante 5 anos a eficácia na prevenção da cárie de um selante à base de resina e outro de ionômero de vidro em primeiros molares permanentes, aplicados por discentes de Odontologia, foi observado que tanto o selante à base de resina quanto o selante de ionômero de vidro podem prevenir efetivamente a ocorrência de lesões de cárie. $O$ estudo mostrou também que o selante à base de resina foi superior ao ionomérico na prevenção de cáries (LIU YJ, et al., 2018). Fato corroborado por Hassan AM e Mohammed SG (2019) que, observando a taxa de retenção de diferentes selantes de fossas e fissuras colocados na superfície oclusal, verificaram um desempenho melhor dos selantes resinosos em comparação ao ionomérico.

Ulusur T, et al. (2012) em pesquisa semelhante mostraram que a retenção dos selantes ionoméricos também foi nitidamente inferior à dos selantes à base de resina, no entanto, o selante ionomérico foi ligeiramente mais eficaz na prevenção de cáries oclusais. Isso se deve ao fato de que selantes resinosos 
convencionais oferecem maior resistência em relação aos selantes ionoméricos, não obstante estes liberam flúor, alcançando ação anticariogênica mesmo com perda física parcial do material (CATÃO MH, et al., 2012).

Porém, outro estudo comparando a retenção, coloração marginal e propriedades cariostáticas entre um selante de ionômero de vidro e um selante à base de resina, em crianças, demonstrou que os dois selantes exibiram taxas de retenção semelhantes em 24 meses. No entanto, a coloração marginal foi menor no grupo do ionômero de vidro, e não foi detectado cárie nos dentes desse grupo. Com base nesses resultados, os autores concluíram que os selantes de ionômero de vidro podem ser uma escolha melhor quando a contaminação salivar é esperada (ANTONSON SA, et al., 2012).

No tocante à aplicação tópica de flúor, uma pesquisa feita com o intuito de observar o comportamento da atividade de manchas brancas ativas e inativas submetidas a tratamento preventivo e conservador (escovação supervisionada quinzenal, escovação diária, aplicações tópicas de flúor, associados a atividades educativas) em 331 prontuários de pacientes de uma escola do município de Maceió-AL, no período de 2011 a 2015, mostrou que $83,33 \%$ das manchas brancas ativas inativaram; apenas $13,85 \%$ das manchas brancas inativas diagnosticadas na primeira consulta cavitaram e os índices indicativos de saúde bucal (cárie, biofilme dental e sangramento gengival) melhoraram significativamente durante o período estudado (Figuras 1, 2 e 3) (MENDONÇA ICG, et al., 2019).

Apesar das evidências científicas apontarem a mínima intervenção como uma técnica segura no tratamento de lesões incipientes de cárie, há no mundo todo diferentes visões entre os dentistas sobre a forma de proceder quando se deparam com cáries dessa natureza, principalmente na face oclusal, e infelizmente muitos decidem intervir cirurgicamente (LASK M, et al., 2019). Na maioria dos casos, o que leva à tomada de decisão pelo procedimento cirúrgico é a presença de uma pigmentação marrom-escura no sulco oclusal, indicação de sulco pigmentado ou inatividade da lesão, ou o fato da sonda se prender na suposta cavidade quando na verdade ele está lidando com uma incompleta coalescência do esmalte na região de fóssulas e fissuras (GORDAN VV, et al., 2010; TAGLIFERRO E, 2019).

Os pacientes liberaram e assinaram o Termo de Consentimento Livre e Esclarecido (TCLE) para fins de publicação científica.

Figura 1 - Cárie inativa sob controle há 16 anos (2002/2018).

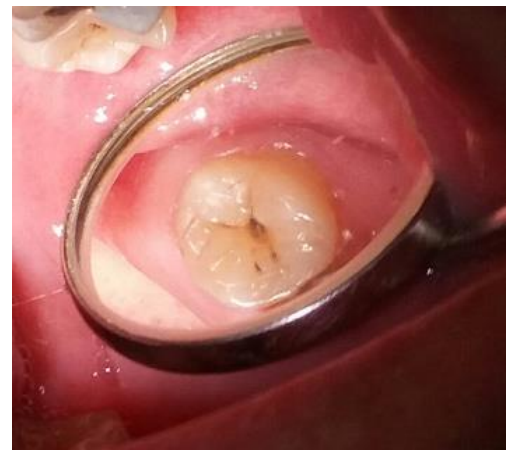

Fonte: Leite LCR, et al., 2020.

Figura 2 - Cárie inativa sob controle há 17 anos (2001/2018).

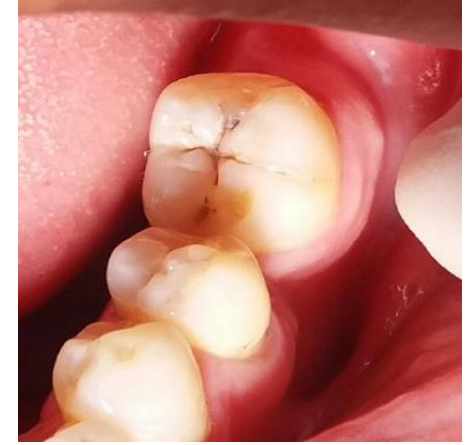

Fonte: Leite LCR, et al., 2020. 
Figura 3 - Cárie inativa sob controle há 12 anos (2006/2018).

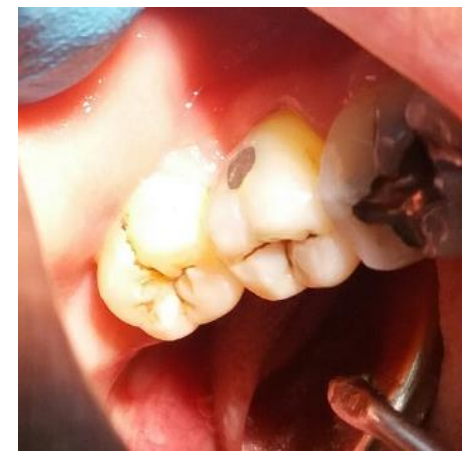

Fonte: Leite LCR, et al., 2020.

\section{Tratamento conservador da cárie proximal}

As cáries proximais, por se alojarem em um local da anatomia dental sem visualização direta, normalmente só é detectada pelos dentistas, no exame visual, quando a cavidade já está instalada e na grande maioria dos casos, já tem tecido dentinário envolvido. A localização também impossibilita a higienização pela escovação, e o fio dental é negligenciado pela maioria dos pacientes. Desse modo, essas lesões são tidas como de progressão rápida e uma intervenção terapêutica é recomendada o mais cedo possível (KIELBASSA AM, et al., 2020).

Como citado no tópico anterior, a radiografia interproximal é um dos principais recursos disponíveis para se obter o diagnóstico da cárie proximal. Já outros autores, encontraram uma preferência entre o uso de explorador e da radiografia interproximal entre os dentistas. No entanto, apesar do uso disseminado, o exame radiográfico parece especialmente adequado para detectar essas lesões quando elas já alcançaram a dentina, entretanto, para lesões incipientes em esmalte, o método apresenta baixa sensibilidade e alta especificidade. A reprodutibilidade das radiografias interproximal varia amplamente e sugerindo que elas podem auxiliar na avaliação das lesões proximais mais avançadas, sendo indicadas quando informações adicionais são necessárias para auxiliar o dentista na tomada de decisão ou no monitoramento de lesões proximais iniciais. (TAGLIAFERRO E, et al., 2019; CARVALHO JC, et al., 2016; GORDAN VV, et al., 2010).

O método de diagnóstico elencado como o mais eficiente é o da transiluminação de luz com comprimento de onda próximo ao infravermelho, conhecido como NIR (near-infrared), pois permite claramente a diferenciação das lesões limitadas ao esmalte daquelas que atingiram a dentina. Os resultados das pesquisas apontam que as radiografias interproximais tendem a subestimar a profundidade da lesão, mesmo se combinadas com a inspeção visual (KIELBASSA AM, et al., 2020).

Em adultos jovens, as superfícies oclusais e proximais mostram experiência de cárie semelhante, porém, as superfícies oclusais são mais gravemente atacadas do que as superfícies proximais quando se considera o grau de gravidade das lesões de cárie, mas a abordagem do tratamento convencional da cárie proximal infere um sacrifício maior de estrutura dental sadia e, aliada a ausência ou falha do manejo preventivo, ainda levará à necessidade de intervenção cirúrgica iniciando uma sequência de ciclos de retratamento com perda crescente de tecido dental duro. O uso de técnicas adesivas possibilitou a utilização de desenhos de preparos menos invasivos, restritos à remoção apenas de tecido cariado, as formas tradicionais de preparação de amálgama retentivo foram abandonadas, enquanto para lesões proximais foram introduzidas preparações em forma de pires. As restaurações de túnel para lesões proximais também foram exploradas, mas provou-se que não tiveram sucesso (CARVALHO JC, et al., 2016).

Porém, um estudo publicado em 2020 derruba essa afirmação. Ele combinou a preparação do túnel interno em combinação com uma infiltração de resina interna e uma abordagem de infiltração externa adicional usando uma resina infiltrante modificada com partículas de nanoprata (AgNP), penetrando melhor nos tecidos, aumentando as áreas de lesão infiltradas, ocluindo e estabilizando adesivamente a porosidade do esmalte desmineralizado, o papel da AgNP nessa associação seria o seu efeito antimicrobiano. Ao final da investigação ex vivo, os autores concluíram que a capacidade da resina infiltrante estudada de se infiltrar na cárie do esmalte proximal não foi afetada negativamente pela adição de AgNP (KIELBASSA AM, et al., 2020). 
Com base nessas observações, parece razoável deduzir que os efeitos antibacterianos da resina infiltrante contendo AgNP devem dificultar a re-formação do biofilme microbiano, aumentando assim a durabilidade da lesão infiltrada sem comprometer suas propriedades mecânicas e impedindo o desenvolvimento de cárie recorrente ou secundária. Com a abordagem de túnel interno apresentada, a combinação de infiltração de resina interna e externa deve resultar em um aumento da estabilização do esmalte desmineralizado e deve evitar qualquer sacrifício de esmalte sadio (KIELBASSA AM, et al., 2020).

A eficácia da aplicação infiltração com resina e recobertas com verniz fluoretado (FV) comparada ao tratamento com FV apenas foi testada em lesões proximais, a experiência concluiu que a infiltração de resina em conjunto com verniz fluoretado parece promissora para controlar a progressão da lesão proximal (EKSTRAND KR, et al., 2010). Esta comprovação é respaldada pela grande maioria das pesquisas que também concluem que esse tratamento é mais eficaz do que a terapia padrão isolada (creme dental com flúor + fio dental). O tratamento não invasivo e microinvasivo tem por objetivo principal retardar a abordagem cirúrgica, enquanto instalam uma barreira no topo (selamento) ou no interior (infiltração) da lesão. Outra pesquisa concluiu que lesões de cárie proximal podem ser tratadas com sucesso com tratamento não operatório, microinvasivo ou restaurador de acordo com o estágio da lesão e a atividade da cárie, o que indica que os casos devem ser considerados individualmente (KIELBASSA AM, et al., 2020; SPLIETH CH, et al., 2020).

Diante de toda complexidade no diagnóstico e tratamento, a prioridade é impedir a progressão e ocorrência da lesão. De modo que a terapia preventiva que tem o intuito de manter a conservação máxima do dente é a escovação com dentifrício fluoretado seguida do fio dental, para que ocorra a inibição no processo de desmineralização do esmalte. Entretanto, esse método só tem funcionalidade e resultado satisfatório mediante o diagnóstico precoce da doença e a completa colaboração do (Figuras 4, 5 e 6).

Figura 4 - Cárie em esmalte entre os elementos 14 e 15. Radiografia inicial.

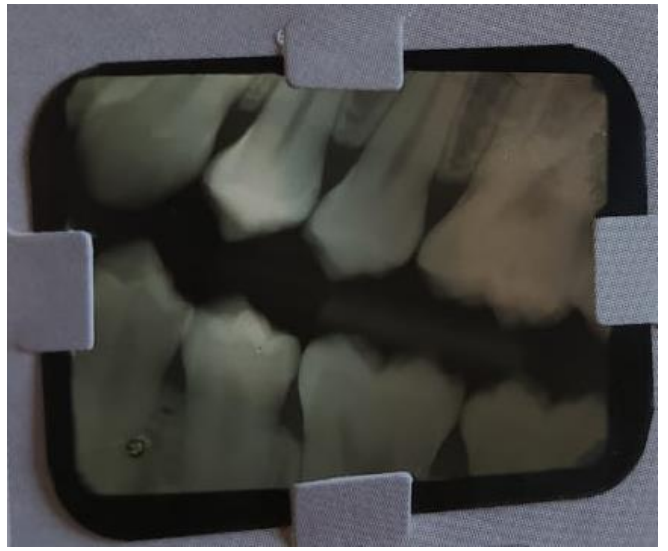

Fonte: Leite LCR, et al., 2020.

Figura 5 - Radiografia de controle 1 ano depois.

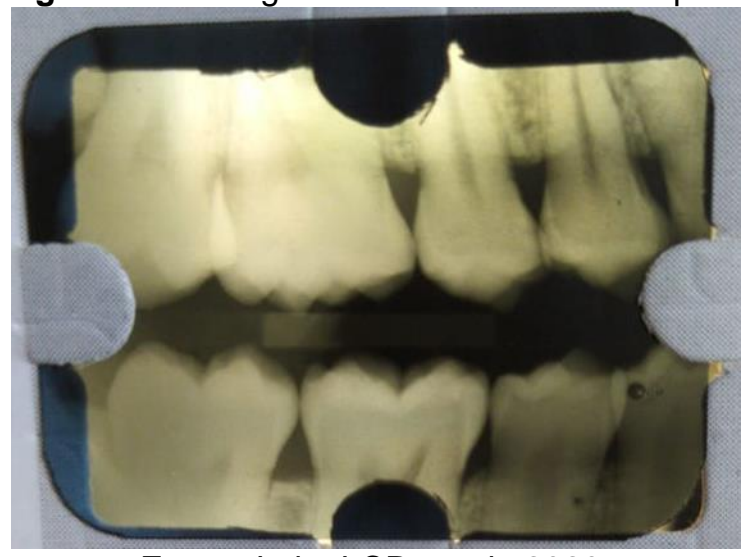

Fonte: Leite LCR, et al., 2020. 
Figura 6 - Radiografia de controle após 4 anos.

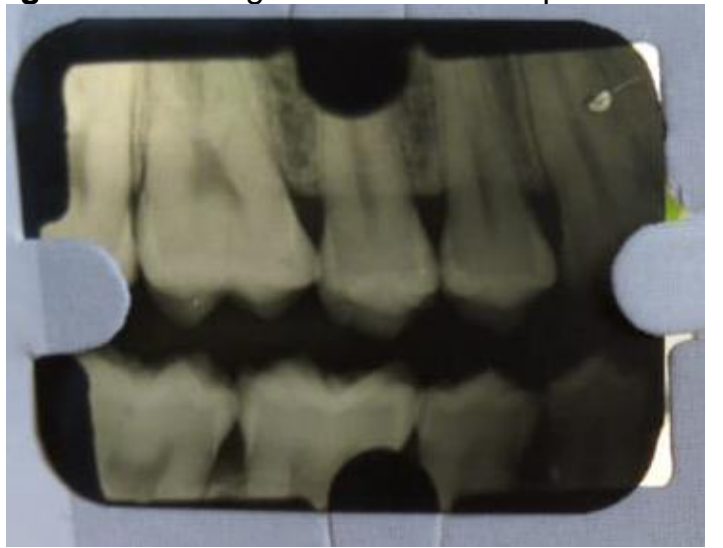

Fonte: Leite LCR, et al., 2020.

\section{CONSIDERAÇÕES FINAIS}

Diante do exposto neste trabalho, e tangenciado cientificamente pela base literária exposta na exordial, a afirmação de que é possível diminuir a chance de desenvolvimento de cárie em dentes recém-erupcionados ou contê-la com a aplicação adequada de selantes de fossas e fissuras, tanto ionoméricos como resinosos, e que o controle eficaz do biofilme aliado a aplicações periódicas de flúor, se sustenta, bem como a adoção de medidas educativas de saúde bucal, são métodos eficientes no controle e tratamento das cáries incipientes em regiões de fossas e fissuras. E que diante de toda complexidade no diagnóstico e tratamento das lesões proximais, a prioridade é impedir a ocorrência e progressão da lesão. Contudo, apesar das evidências em contrário, ainda há uma resistência muito grande da classe odontológica em adotar medidas minimamente invasivas.

\section{REFERÊNCIAS}

1. ANTONSON SA, et al. Twenty-four month clinical evaluation of fissure sealants on partially erupted permanent first molars: glass ionomer versus resin-based sealant. J Am Dent Assoc. 2012; 143(2): 115-22.

2. BATISTA MJ, et al. Classificação das perdas dentárias: fatores associados a uma nova medida em uma população de adultos. Ciênc. saúde coletiva, 2015; 20(9): 2825-2835.

3. CARVALHO JC, et al. Occlusal Caries: Biological Approach for Its Diagnosis and Management. Caries Res. 2016; 50(6): 527-542.

4. CATÃO MHCV, et al. Importância do selamento de fóssulas e fissuras na prevenção da cárie dental: revisão de literatura. HU Revista, Juiz de Fora, 2012; 38(1 e 2): 103-109.

5. CHESTNUTT IG, et al. Fissure Seal or Fluoride Varnish? A Randomized Trial of Relative Effectiveness. J Dent Res. 2017; 96(7): 754-761.

6. EKSTRAND KR, et al. Treatment of proximal superficial caries lesions on primary molar teeth with resin infiltration and fluoride varnish versus fluoride varnish only: efficacy after 1 year. Caries Res. 2010; 44(1): 41-46.

7. FEJERSKOV O, KIDD E. Cárie Dentária: a doença e seu tratamento clínico. São Paulo. Ed. Santos, 2007.

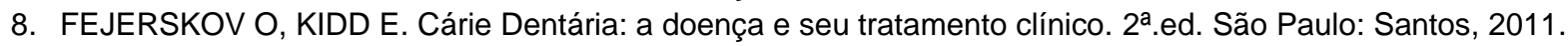

9. FEJERSKOV O, et al. Dental Caries: The Disease and Its Clinical Management. 3rd Ed. Oxford (UK): Wiley Blackwell, 2015.

10. GAO SS, et al. Caries remineralisation and arresting effect in children by professionally applied fluoride treatment - a systematic review. BMC Oral Health, 2016; 16: 12.

11. GORDAN VV, et al. Dental Practice-Based Research Network Collaborative Group. Restorative treatment thresholds for occlusal primary caries among dentists in the dental practice-based research network. J Am Dent Assoc. 2010; 141(2): 171-84.

12. HASSAN AM, MOHAMMED SG. Effectiveness of Seven Types of Sealants: Retention after One Year. Int J Clin Pediatr Dent. 2019; 12(2): 96-100.

13. KIELBASSA AM, et al. Investigação ex vivo sobre abordagem de túnel interno / infiltração de resina interna e infiltração externa de resina modificada por nanoprata de cáries proximais excedendo na dentina. PLoS ONE, $2020 ; 15$ (1): e0228249. 
14. LASKE M, et al. Minimally Invasive Intervention for Primary Caries Lesions: Are Dentists Implementing This Concept? Caries Res. 2019; 53(2): 204-216.

15. LIU YJ, et al. Caries prevention effectiveness of aresin based sealant and a glass ionomer sealants: a report of 5-yearfollow-up. Zhonghua Kou Qiang Yi Xue Za Zhi, 2018; 53(7): 437-442.

16. MAKHIJA SK, et al. National Dental PBRNCollaborative Group. Dentists' decision strategies for suspicious occlusal caries lesions in a National Dental PBRN study. J Dent. 2018; 69: 83-87.

17. MENDONÇA ICG, et al. Monitoramento da atividade de lesões iniciais de cárie em alunos de uma escola do município de Maceió-AL após tratamento preventivo conservador. Revista Eletrônica Acervo Saúde, 2019; 20 : e584.

18. NYVAD B, BAELUM V. Nyvad Criteria for Caries Lesion Activity and Severity Assessment: A Validated Approach for Clinical Management and Research. Caries Res. 2018; 52(5): 397-405.

19. NYVAD B, et al. Diagnóstico tátil-visual da cárie. In: Kidd E, Fejerskov O. Cárie Dentária: A Doença e seu Tratamento Clínico. 2ª Ed. Tradução: Rossetti PHO. São Paulo: Santos, 2011; 50-68.

20. PETRIK JÁ, et al. Avaliação da condição dos primeiros molares permanentes em crianças e adolescentes assistidas em um projeto social. Arquivos do Mudi, 2020; 24(1): 1-11.

21. RIBEIRO AA. Biological Features of Dental Caries. JSM Dent. 2016; (3): 1065-70.

22. SILVA EL, et al. Abordagem Terapêutica em Lesões Cariosas: Quando e Como Tratar. Revista Brasileira de Ciências da Saúde, 2017; 21(2): 173-180.

23. SILVA BP, et al. Current trends in the conservative treatment of deep-caries lesion with risk of pulp exposure. Revista Brasileira de Odontologia, 2018; 75:1.

24. SOARES GG, et al. Métodos de detecção de cárie. Rev. Bras. Odontol. 2012; 69(1): 84-89.

25. SPLIETH CH, et al. How to intervene in the caries process: proximal caries in adolescents and adults-a systematic review and meta-analysis. Clin Oral Investig. 2020; 24(5): 1623-1636.

26. TUMENAS I, et al. Odontologia Minimamente Invasiva. Rev. Assoc. Paul. Cir. Dent. 2014; 68(4): $283-295$.

27. TAGLIAFERRO EP, et al. Caries Diagnosis in Dental Practices: Results From Dentists in a Brazilian Community. Oper Dent. 2019; 44(1): E23-E31.

28. TAGLIAFERRO EP, et al. [Cost-effectiveness analysis of preventive methods for occlusal surface according to caries risk: results of a controlled clinical trial]. Cad Saúde Pública, 2013; 29 Suppl 1: S121-130.

29. ULUSUR T, et al. The success rates of a glass ionomer cement and a resin-based fissure sealant placed by fifth-year undergraduate dental students. Eur Arch Paediatr Dent. 2012; 13(2): 94-97. 